\title{
Joint DAMA-TCP protocol optimization through multiple cross layer interactions in DVB RCS scenario
}

\author{
M. Luglio, F. Zampognaro \\ Electronics Engineering Department \\ University of Rome "Tor Vergata" \\ Rome, Italy
}

\author{
T. Morell, F. Vieira \\ Telecommunications and System Engineering Department \\ University of Barcelona "UaB" \\ Barcelona, Spain
}

\begin{abstract}
Two aspects of DVB-RCS standard can worsen performance of TCP data connections: DAMA access scheme, since it introduces additional and variable delay to the already significant propagation delay and the adoption of Adaptive Coding on the return link to maximize bandwidth efficiency to face variable weather conditions, because it results in variable bandwidth allocation. Both aspects can severely impact TCP performance, especially for what concerns flow adaptation to varying channel conditions and channel usage efficiency. To optimize performance, in this paper cross-layer signaling among transport, MAC and physical layers of a DVB-RCS system is addressed. In particular MAC-TCP cross-layer is analyzed through the use of NS2 network simulator, showing the possible benefit in a DVB-RCS scenario.
\end{abstract}

Keywords-AC, Cross-layer, NS2, DVB-RCS, DAMA

\section{INTRODUCTION}

The Digital Video Broadcasting - Return Channel over Satellite (DVB-RCS) standard [1] assumes a significant importance when a space segment is included in the path for communications based on the TCP-IP protocol (as in the case of multimedia applications such as web browsing, mailing services, file transfer applications, etc.). The standard basically defines layers 1 and 2 of the OSI protocol stack.

In this work we aim to contribute both with system design and analysis on a cross-layer enabled DVB-RCS system. First we introduce a proper cross-layer Dynamic Bandwidth Allocation (DBA) algorithm that supports the Demand Assignment Multiple Access (DAMA) mechanism envisaged in DVB-RCS. Secondly a Medium Access Control (MAC)TCP interaction is proposed to counteract the undesirable effects introduced by changes in the perceived link quality (for example in case of a rain fading event) to TCP performance. Cross layer in such a critical environment can bring considerable benefits at the price of violating OSI layering. Cross-layer in particular will be explicit between MAC-PHY and DBA and between MAC and TCP, while DBA and TCP will have no direct signaling. In that way the problem can be split in two independent parts, still mutually interfering, which will be discussed in the following sections.
Than we developed a DVB-RCS system simulator utilizing Network Simulator-2 (NS-2). It is used to test the benefits reported with the cross-layer mechanism related to transport layer.

The paper is so organized: in section II DVB-RCS scenario considered and cross-layer mechanisms are introduced; in section III and IV DBA and TCP specific aspects are addressed; in $\mathrm{V}$ the simulations performed are described and results are commented; finally conclusions are drawn in VI.

\section{REFERENCE SCENARIO}

The reference scenario consists of a transparent satellite star network adopting the DVB-RCS technology, shown in its main block elements in Figure 1. We assume that each DVB-RCS Terminal (RCST) works with Differentiated Services (DiffServ) and that TCP traffic is going from RCSTs to the NCC. IP queues are then mapped to MAC-layer queues depending on the particular Quality of Service (QoS) policies.

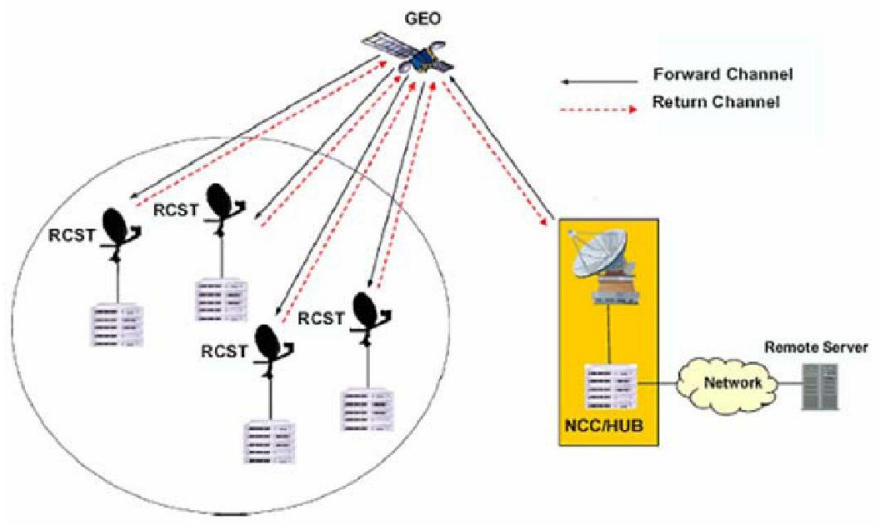

Figure 1. DVB-RCS Scenario

In the presence of information to transmit at MAC queues, RCSTs react by requesting capacity to the Network Control Center (NCC), which is attached to a terrestrial Gateway, using Satellite Access Control (SAC) messages. Emitted capacity requests respond basically to three main types: 
- Constant Rate Assignment (CRA) - the terminal requests a constant rate to transmit during all the time.

- Rate Based Dynamic Capacity (RBDC) - transmission rate is requested and it is active until it is updated or timed out. Compared to CRA, this request type allows for statistical multiplexing of users and the possibility to use bandwidth efficiently.

- Volume Based Dynamic Capacity (VBDC): it requests for transmitting a certain volume of data according to the actual needs and the past requests and can increase bandwidth efficiency even more (no constant rate needed).

When the requests from all RCSTs are available at the NCC, it arranges the multiplexed transmission according to Multi Frequency - Time Division Multiple Access (MFTDMA) discipline as defined for DVB-RCS. In short, it assigns the time and frequency coordinates where a given RCST can transmit its data, organized in timeslots, along the Superframe (SF). It also establishes the shape of the timeslots in the Terminal Burst Time Plan (TBTP), broadcasted to all RCSTs. The current version of the DVB-RCS standard actually leaves open details on how to build the TBTP.

An example of a general DVB-RCS system is depicted in Figure 2 in more details, with inner elements of a RCST and the messages exchanged. The proposed cross layer messaging is represented by dotted arrows and includes both cross layer information crossing the space segment and remaining inside RCST.

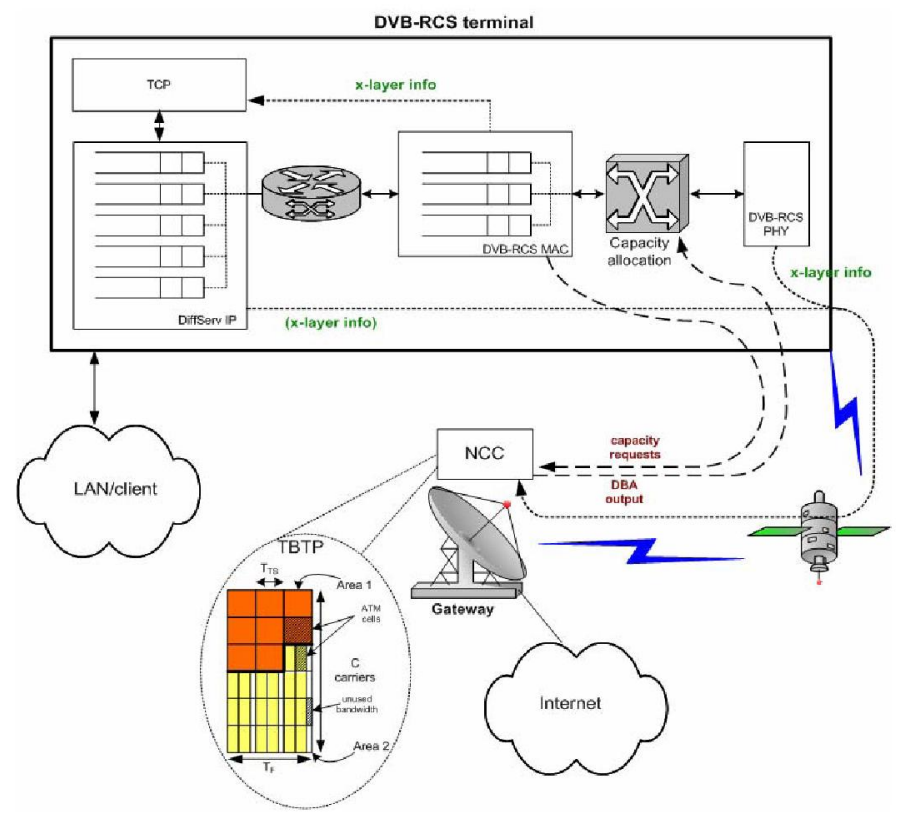

Figure 2. DVB-RCS, TCP and cross layer

\section{DBA ALGORITHM AND CROSS LAYER}

In DVB-S2, terminals can adapt their transmission scheme according to the experienced link quality changing both coding and modulation schemes (Adaptive Coding and Modulation, $\mathrm{ACM}$ ). In the return link of a DVB-RCS system a RCST is only allowed to adjust coding rate (Adaptive Coding, AC). Thanks to $\mathrm{AC}$, RCSTs can set up a reliable transmission of information even in the case of a temporary bad link performance.

It is worth to take the AC schemes of each RCST into account at the NCC when computing the TBTP, establishing the proposed cross-layer interaction. A further optional crosslayer interaction, with the IP layer, could aim to distinguish traffic types that are requested under the same capacity request but have different QoS policies. For example, let's suppose to have a File Transfer Protocol and a web browsing application both using VBDC to request capacity over different RCSTs. It would be desirable to prioritize web browsing over FTP in the computation of the TBTP and assign to the terminal performing Web browsing more capacity.

We assume that the available bandwidth is divided into a fixed number of subcarriers and we group together the carriers with the same bandwidth. Depending on the terminal equipment, Service Level Agreement, etc., RCSTs are distributed among those groups.

DBA implementation is then split into several smaller subproblems, to allocate the RCSTs in each subgroup. To allocate resources to terminals that transmit using the same subcarrier bandwidth we propose to consider common timeslots to all RCSTs regardless the coding rate they transmit with. Then, when a timeslot is allocated to a certain RCST, it transmits as much MAC layer units (e.g. ATM cells or MPEG containers) as possible inside the timeslot. For a given subcarrier bandwidth, the time duration of the transmission of a MAC layer unit (a fixed amount of information) depends on the coding rate the RCST uses: for high coding rates less transmission time is required. In TBTP of Figure 2, for example, RCSTs are grouped under two different coding rates under the labels Area 1 and Area 2.

Note that the definition of a common timeslot allows reduced signaling as far as we only need to define, at the beginning of the subcarrier, the shape and the number of timeslots. The general approach, where the position and shape of all timeslots in the superframe is explicitly signaled, requires pretty higher signaling rates.

Once the timeslot duration is fixed, they are allocated to RCSTs according to a Network Utility Maximization formulation [2] particularized to the case of DVB-RCS for the most general case:

$$
\begin{array}{lc}
\max _{\left\{x_{i, j}\right\}} \quad \prod_{i, j}\left(x_{i, j} \cdot K_{i}\right)^{p_{i, j}} \\
\text { s.t. } & \sum_{i, j} x_{i, j} \leq P \\
& {\left[\frac{m_{i, j}}{K_{i}}\right\rceil \leq x_{i, j} \leq\left\lceil\frac{d_{i, j}}{K_{i}}\right]}
\end{array}
$$

where $x_{i, j}, m_{i, j}$ and $d_{i, j}$ are the number of MAC layer units assigned to the $i^{\text {th }} \mathrm{RCST}$ in the $\mathrm{j}^{\text {th }}$ connection, the minimum guaranteed number and the requested number of MAC layer 
units, respectively. $\mathrm{P}$ is the available number of timeslots and $K_{i}$ is the number of MAC layer units that the $\mathrm{i}^{\text {th }} \mathrm{RCST}$ can transmit in a timeslot. Finally, $p_{i, j}$ is the priority of the $\mathrm{j}^{\text {th }}$ connection of the $i^{\text {th }}$ RCST.

If traffic priorities information is not available as input to the DBA algorithm, (1) is simplified as the $\mathrm{j}$ index can be omitted. In this case $p_{i}$ represents a single value of priority assigned to each terminal $x_{i}, m_{i}$ and $p_{i}$ are respectively the assigned, minimum guaranteed and the requested number of MAC layer units per terminal. The previous formulation corresponds to an asymmetric fair distribution of resources (see the definition of proportional fairness in [3]). Note that an adequate adjustment of priorities allows balancing the distribution and making the DAMA as transparent as possible to the QoS levels defined in DiffServ. Cross-layer information necessary for the algorithm can be signaled using the Channel ID field of SAC messages, which is actually unused in transparent satellite networks. Note that $K_{i}$ depends on the coding rate the RCST is transmitting with, and it is a parameter of the physical layer. For a given timeslot duration, the higher the coding rate, the higher the number of MAC layer units inside a timeslot. It is possible to easily tune the timeslot duration to maximize (1) function of the timeslot duration or, in other words, to better exploit the superframe available bandwidth. We refer the interested reader to the authors' work in [4].

Finally, it is necessary to compute the solution of (1) in the most efficient way since only few milliseconds are available for the NCC to update the TBTP table. Therefore, computation time may be, in huge networks, a limiting factor (of the number of users). For the sake of conciseness, we do not discuss here on computational efficiency and adequate procedures and we refer the interested reader to the authors' work in [5].

\section{TCP AND CROSS LAYER}

Standard TCP connections performance over DVB-RCS suffers of combined effect of DAMA and AC on both data transfer directions and in particular for the data transfer from RCSTs to NCC. In fact, the proposed DBA algorithm aims to fairly use the return channel and, in the event of $\mathrm{AC}$ variations, to introduce variations in the perceived channel capacity with regard to TCP. As already introduced, there will be no explicit cross-layer signaling between DBA and transport layer adopting TCP, but TCP will be designed to better cope with underlying variations with only information derived from MAC layer queues.

The proposed Transport-MAC cross-layer interaction is based on forcing TCP sending window [6] to a given value elaborated when sudden increase/decrease of the sender queue size is experienced. In fact, if the sender queue suddenly changes this can be due to a variation of the available capacity at MAC layer.

The sender window will be tuned taking into account the congestion window ( $c$ wnd) value, the advertised window $(a d v$ wnd) value announced by the receiver of the connection at the NCC side and an estimation of the optimal value using cross layer information (xlayer_wnd), taking into account the value of the window before the variation and the entity of the variation itself according to:

$$
\text { send_wnd }=\min \{c w n d, \text { adv_wnd, xlayer_wnd }\}
$$

where:

$$
\begin{gathered}
\text { xlayer_wnd }=\text { current_wnd }+\alpha\left(q u e u e \_s i z e[n-1]-\right. \\
\text { queue_size }[n)] / p k t \_ \text {size }
\end{gathered}
$$

The $\alpha$ parameter is a weighting factor needed to correctly balance the effect of window adjusting. Equation (3) should be applied only if the queue variation is above a fixed threshold and for longer than a defined time interval, needed to avoid fast fluctuations of the current transmission window. The final goal of this cross-layer mechanism is to perform some sort of explicit flow control and avoid sender queue overflow.

\section{Simulation}

With regard to the MAC-transport cross-layer, simulations have been performed in a DVB-RCS scenario. The NS2 event driven network simulator [7] has been used, having improved it with some custom code.

\section{A. DAMA in NS2}

A preliminary functional DAMA allocation scheme has been implemented as NS2 core functionality, including the satellite terminals capacity request algorithms according to [8]. For the current simulation campaign we assumed a scenario with only one terminal, avoiding competition in the assignment of frames, but still representative of access delay of a basic DAMA access scheme. The variable access delay introduced by the NS2 implementation of DAMA is clearly visible from oscillations of a TCP New Reno connection RTT in Figure 3, on a terminal using a mix of CRA, RBDC and VBDC requests. Such oscillations are comparable to the ones shown in [8]. At steady state TCP New Reno, without sending window constraints, increases its transmission rate until, at time $105 \mathrm{~s}$, it saturate the full return link capacity, and RTT increases constantly as consequence.

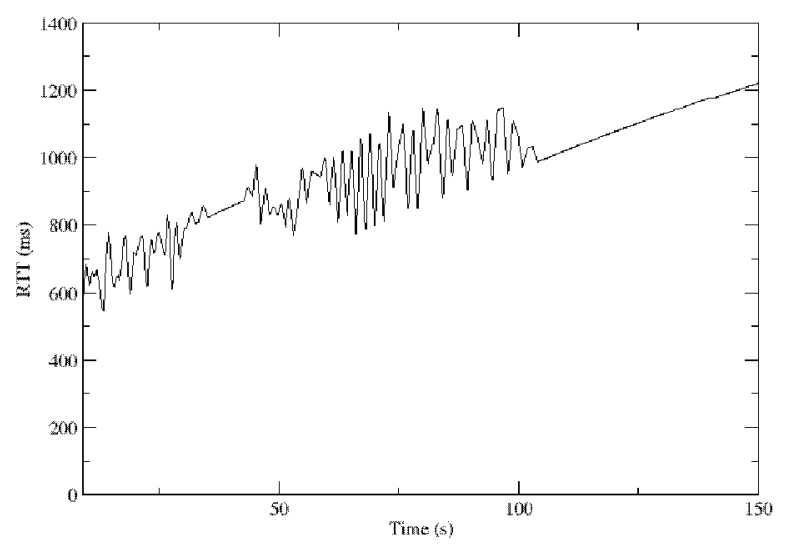

Figure 3. Variable access delay introduced by DAMA to perceived RTT

A DBA scheme compliant with that proposed in section III is in process of implementation in NS2, and will be object of a following article describing a complete interaction analysis of 
DAMA and TCP file transfers, using the cross-layer mechanism proposed and with multiple RCSTs.

\section{B. Cross layer implementation}

The proposed cross layer mechanism has been implemented on NS2 allowing TCP New Reno sender to send each time it is allowed only a given amount of packets following (2). In order to evaluate that equation, it is needed as input a variable representative of the instantaneous queue usage value of the terminal (cross-layer). Equation (2) is applied, with the limit of using a fixed value for adv wnd: a dynamic handling of $a d v$ wnd in fact is not implemented in the current version of NS2.

\section{Simulation set up and parameters}

TCP data flow, from the RCST to the NCC, uses an overall capacity of $2.1 \mathrm{Mbit} / \mathrm{s}$. The forward channel capacity, set to 2 $\mathrm{Mbit} / \mathrm{s}$, is only used to reply relatively small ACKs to the RCST. Requests are issued according to a mixed request scheme of CRA, RBDC and VBDC. All simulated links and nodes buffers are designed large enough so that packet loss is avoided. In this simulation at $200 \mathrm{~s}$ a $17 \%$ capacity reduction on the return channel has been applied, in order to trigger a sudden variation comparable to an $\mathrm{AC}$ event.

The adv wnd parameter has been set to a pre-defined optimal value of 167 packets, tuned according to the link bandwidth-delay product. Slow start threshold (ssthresh) parameter has been set to a relatively high value of 60 packets, in order to compensate for large RTT experienced on the satellite environment and let TCP window to grow faster in the initial slow start phase. Without losses and with the $a d v$ wnd correctly set, TCP New Reno is able to reach after about $\overline{1} 10 \mathrm{~s}$ the maximum channel capacity with an equilibrium queue usage of about 92 Kbytes

\section{Comments on results}

Figure 4 shows the throughput of the TCP connection: after more than $100 \mathrm{~s}$, using TCP New-Reno, the maximum throughput is reached and remains constant, thanks to the preconfigured optimal $a d v$ wnd. At $200 \mathrm{~s}$ is clearly visible the effect of bandwidth reduction.

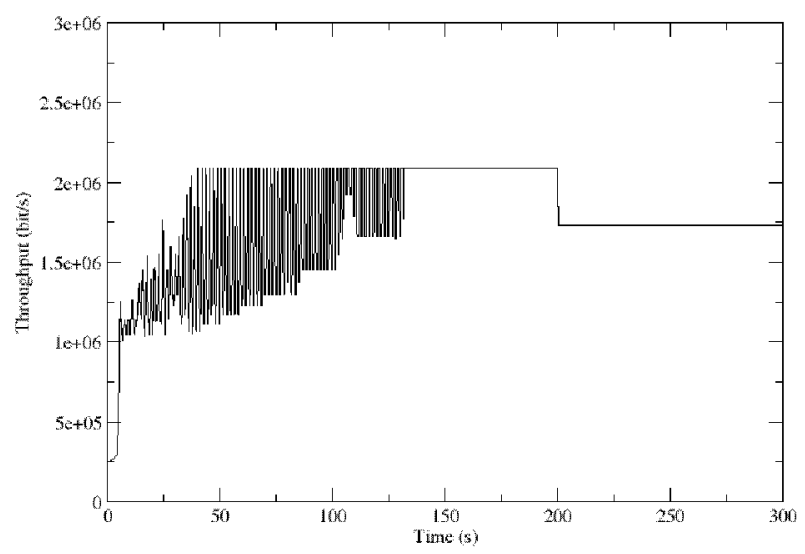

Figure 4. Bandwidth variation on MAC capacity variations
At $200 \mathrm{~s}$ the perceived RTT is suddenly increasing, due to an overfill of the queue, as seen in Figure 5 . The queue in fact has less capacity to send its packets to lower layers and, since the sending rate remains constant, it stabilize to a newer higher equilibrium value of 120 Kbytes (see Figure 6).

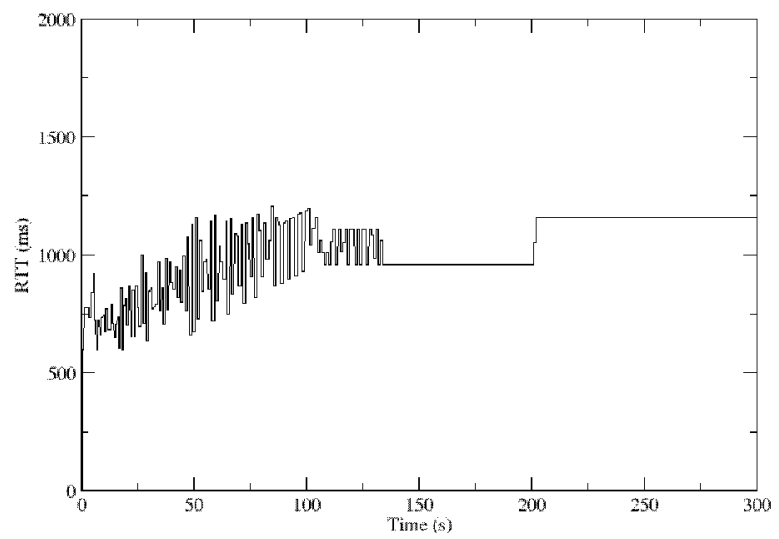

Figure 5. RTT variation on MAC capacity variations

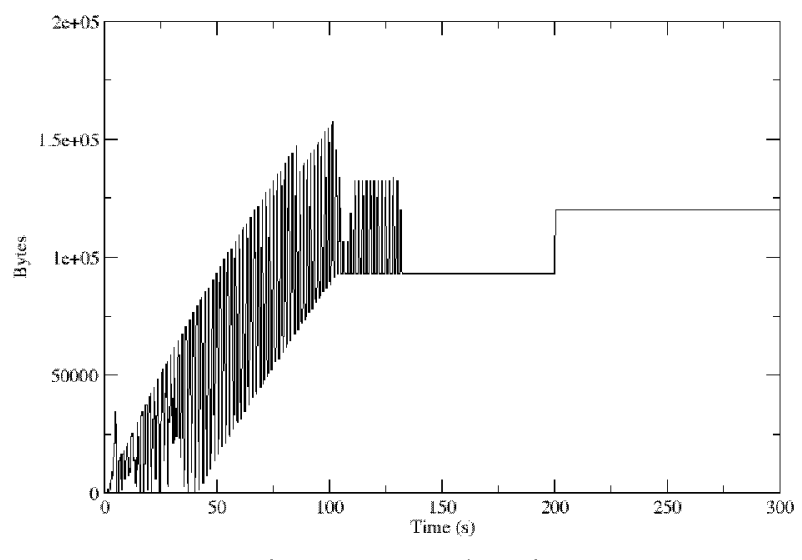

Figure 6. Queue dynamics

To enable the cross layer mechanism, (3) has been applied in a way that there is a counter response to the queue inflation with a sending window decrease, using an $\alpha$ value of 1.5. As depicted in Figure 7, the queue usage after the cross layer action is decreasing and stabilizes with few oscillations. In response to the capacity reduction and the cross layer action, the perceived RTT is maintained at the original value measured before the capacity variation, as shown in figure 8 .

A direct comparison of Figure 5 and 8 is useful to understand the benefit of the cross layer approach, in terms of RTT. 


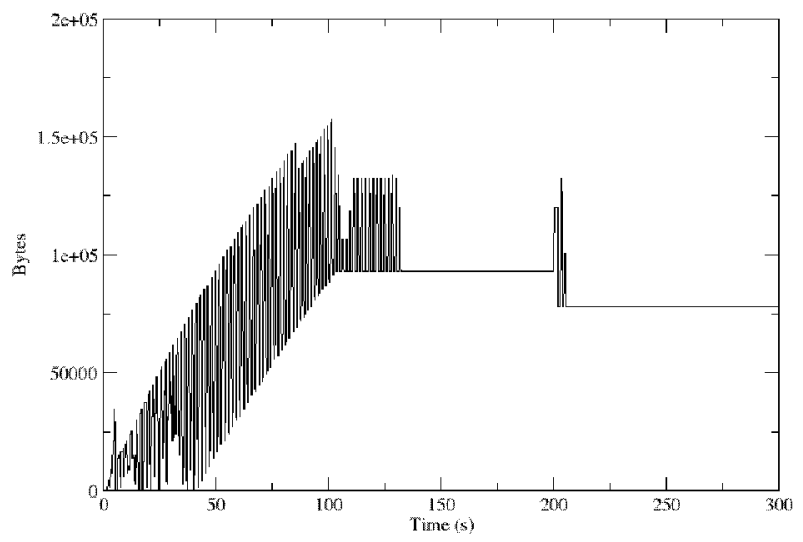

Figure 7. Queue dynamics with cross-layer

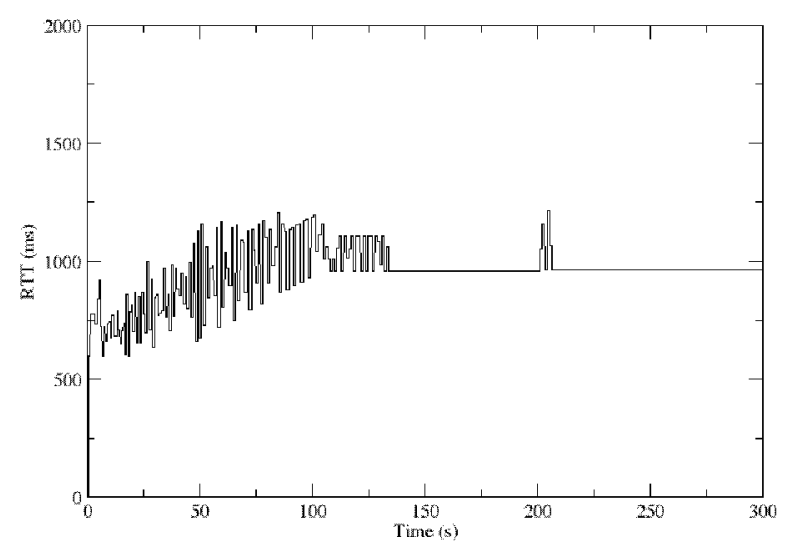

Figure 8. RTT variations with cross-layer

An observed counter effect is a small bandwidth loss in the transient at $200 \mathrm{~s}$, shown in the detailed zoom of Figure 9. In fact the reaction time of the cross-layer mechanism and the sudden variation of the transmission window lead to a small and temporary underutilization of the channel rate resulting in a throughput reaching a value lower than the maximum allowed.

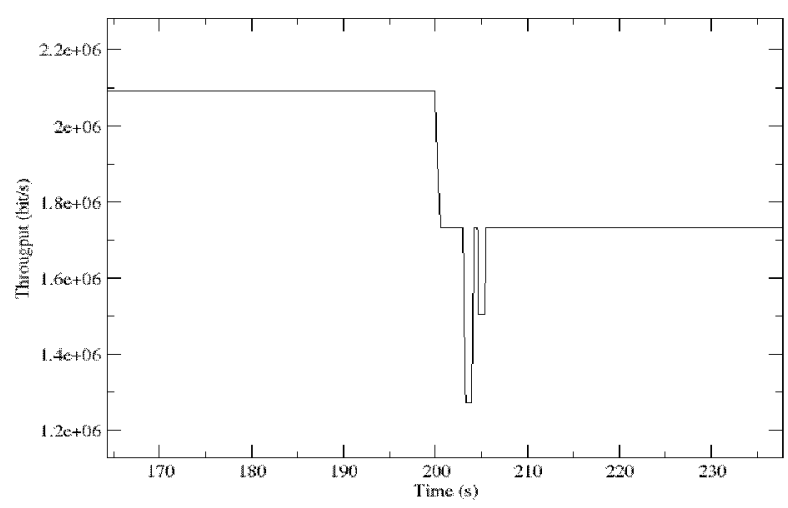

Figure 9. Bandwidth loss with cross layer

\section{CONCLUSION}

To enhance data transmission efficiency in the paper we have proposed two cross-layer mechanisms: i) a novel crosslayer framework to optimize the Dynamic Bandwidth Assignment (DBA) in the DAMA (Demand Assignment Multiple Access) of the DVB-RCS and ii) an optimized crosslayer TCP using MAC-layer information. No explicit interaction between DBA and transport layer adopting TCP is proposed, but whenever the implementation of DBA or AC is delivering system capacity variations, consequences at $\mathrm{MAC}$ layer will trigger cross-layer messages from MAC to transport layer in order to optimize the TCP data transfer in terms of RTT and throughput. The latter mechanism has been analyzed through simulation and has shown encouraging preliminary results.

\section{ACKNOWLEDGMENT}

This paper is part of the results of a Joint Activity of University of Rome "Tor Vergata" and University of Barcelona "UaB" in the frame of the European Satellite Network of Excellence [9]. The authors gratefully acknowledge SatNEX for its support and communication help.

\section{REFERENCES}

[1] ETSI, "Digital Video Broadcasting (DVB); Interaction Channel for Satellite Distribution Systems", ETSI EN 301 790, Apr 2005.

[2] D.P. Palomar and M. Chiang, "Alternative Decompositions for Distributed Maximization of Network Utility: Framework and Applications", in Proc. IEEE Infocom, Barcelona, Spain, Apr. 2006.

[3] F.P. Kelly, A. Maulloo and D. Tan, "Rate control for communication networks: shadow prices, proportional fairness and stability", Journal of Operations Research Society, Vol. 49, No. 3, pp. 518-528, Apr. 2004.

[4] A. Morell, G. Seco-Granados and M.A. Vázquez-Castro, "Joint Time Slot Optimization and Fair Bandwidth Allocation for DVB-RCS Systems", in proc. IEEE GLOBECOM'06, San Francisco, Nov. 2006.

[5] A. Morell, G. Seco-Granados and M.A. Vázquez-Castro, "Computationally Efficient Cross-Layer Algorithm for Fair Dynamic Bandwidth Allocation", to appear in proc. of IEEE ICCCN'07, Honolulu, Aug. 2007.

[6] W. Stevens, "TCP/IP Illustrated vol.1", Addison Wesley, MA, USA, 1994.

[7] K. Fall and K. Varadhan. "The NS Manual", http://www.isi.edu/nsnam/, 2002

[8] C. Roseti and E.Kristiansen, "TCP behaviour in a DVB-RCS environment". In Proceedings 24th AIAA International Communications Satellite Systems Conference (ICSSC), San Diego, 2006.

[9] European Satellite Communications Network of Excellence (SatNEx), Phase II, http://www.satnex.org 